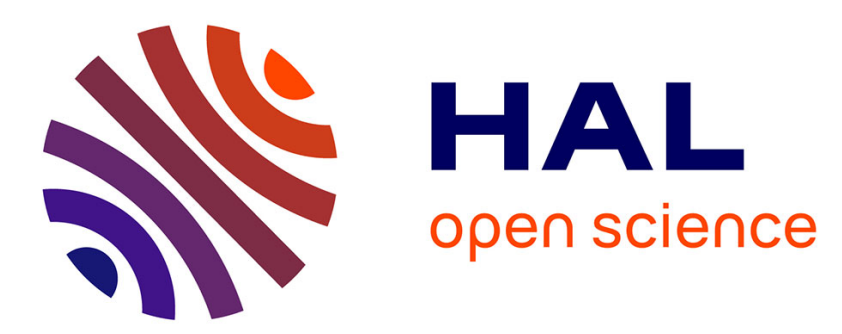

\title{
Éclatement du régime de l'autorité et dérégulation des relations de pouvoir : la Province dominicaine de France après Mai 68
}

\author{
Yann Raison Du Cleuziou
}

\section{To cite this version:}

Yann Raison Du Cleuziou. Éclatement du régime de l'autorité et dérégulation des relations de pouvoir : la Province dominicaine de France après Mai 68. Histoire@Politique: revue du Centre d'histoire de Sciences Po, 2012, 18, pp.97-114. 10.3917/hp.018.0097 . halshs-00763717

\section{HAL Id: halshs-00763717 https://shs.hal.science/halshs-00763717}

Submitted on 19 Jan 2022

HAL is a multi-disciplinary open access archive for the deposit and dissemination of scientific research documents, whether they are published or not. The documents may come from teaching and research institutions in France or abroad, or from public or private research centers.
L'archive ouverte pluridisciplinaire HAL, est destinée au dépôt et à la diffusion de documents scientifiques de niveau recherche, publiés ou non, émanant des établissements d'enseignement et de recherche français ou étrangers, des laboratoires publics ou privés. 
Yann Raison du Cleuziou, «Éclatement du régime de l'autorité et dérégulation des relations de pouvoir : la Province dominicaine de France après Mai 68 », Histoire@Politique. Politique, culture, société, $n^{\circ} 18$, septembre-décembre 2012 [en ligne, www.histoire-politique.fr]

\title{
Éclatement du régime de l'autorité et dérégulation des relations de pouvoir : la Province dominicaine de France après Mai 68
}

\author{
Yann Raison du Cleuziou
}

La vague de contestation qui frappe les institutions catholiques après Mai 68 est connue ${ }^{1}$. Le catholicisme se décompose alors en de multiples factions ouvertement concurrentes pour définir le christianisme légitime ${ }^{2}$. Le clergé est au coeur de cette crise $^{3}$. Au sein du clergé séculier, le mouvement Échanges et Dialogue invente une forme inédite de contestation du sacerdoce ${ }^{4}$. Ses militants aspirent à faire des prêtres des hommes normaux que plus rien ne distingue. Le clergé régulier est inégalement atteint par la contestation. D'un côté l'abbaye de Boquen sous la gouverne de Dom Bernard Besret s'aventure dans des expériences communautaires novatrices ${ }^{5}$; d'un autre côté les bénédictins de Solesmes ne s'écartent pas de la ligne romaine et réforment le strict nécessaire. Les dominicains de la Province de France sont quant à eux profondément divisés : les partisans d'un profond renouvellement des formes de la vie religieuse s'affrontent avec ceux qui privilégient une évolution mesurée de la vie conventuelle et privilégient la continuité des formes traditionnelles.

Composée de près de cinq cent cinquante religieux à la fin des années 1960, la Province dominicaine de France occupe une position importante dans l'Église de France. Elle bénéficie du prestige de ses théologiens comme Yves Congar ou MarieDominique Chenu, de ses missionnaires comme le père de Beaureceuil ou encore de ses prédicateurs comme Ambroise-Marie Carré. Les éditions du Cerf créées et dirigées par les dominicains sont un pivot de la vie intellectuelle catholique. Au-delà de l'hexagone, la Province de France occupe également une place de choix dans

\footnotetext{
${ }^{1}$ Gérard Cholvy, Yves-Marie Hilaire, Histoire religieuse de la France contemporaine, tome 3, Toulouse, Privat, 1988, chap. 7. ; Denis Pelletier, La crise catholique. Religion, société, politique, Paris, Payot, 2002.

2 Étienne Fouilloux, Les chrétiens français entre guerre d’Algérie et Mai 1968, Paris, DDB, 2008, p. 337-343.

${ }^{3}$ Martine Sevegrand, Vers une église sans prêtres. La crise du clergé séculier en France (1945-1978), Rennes, PUR, 2004.

${ }^{4}$ Denis Pelletier, « Des prêtres contestataires dans la France des années 68 », dans Dominique IognaPrat et Gilles Veinstein (dir.), Histoires des hommes de Dieu dans l'islam et le christianisme, Paris, Flammarion, 2003.

5 Yvon Tranvouez, «L'abbaye de Boquen : d'une expérience de réforme monastique à un haut lieu de l'esprit de Mai 68 », dans Alain Gérard (dir.), Des curés aux entrepreneurs. La Vendée au XX ${ }^{\mathrm{e}}$ siècle, La Roche-sur-Yon, Centre vendéen de recherches historiques, 2004, p. 499-512.
} 
Yann Raison du Cleuziou, «Éclatement du régime de l'autorité et dérégulation des relations de pouvoir : la Province dominicaine de France après Mai 68 », Histoire@Politique. Politique, culture, société, $n^{\circ} 18$, septembre-décembre 2012 [en ligne, www.histoire-politique.fr]

l'ordre dominicain car elle se place à la tête du courant réformateur ${ }^{6}$. Le dépouillement de ses archives permet d'apporter un éclairage nouveau sur les années 1960 et 1970, trop souvent connues à travers les seuls imprimés et les témoignages. Les correspondances entre religieux, les papiers de l'administration provinciale où sont archivées les minutes des conseils qui décident des orientations collectives, et les dossiers des différentes commissions en charge des réformes, montrent à quel point l'exercice de l'autorité va provoquer des drames intimes, certains religieux trouvant nécessaire de désobéir à leur supérieur pour rester fidèles à leurs vœeux.

Le contexte de l'année 1968 doit être décrit avec minutie pour comprendre le conflit autour de l'autorité provinciale qui s'y est engagée. Aux mois de mai et de juin 1968, le couvent d'étude du Saulchoir, où les jeunes religieux dominicains suivent leurs sept années d'études, est traversé par la contestation. Les frères étudiants se réunissent en assemblées générales et dénoncent le caractère suranné et peu évangélique des formes religieuses auxquelles ils doivent se conformer. Nicolas Rettenbach, qui est titulaire depuis 1967 de la charge de provincial, c'est-à-dire de supérieur de la Province, et la jeune équipe de lecteurs (enseignants en théologie) nommée en 1965 autour de Claude Geffré, encouragent le mouvement étudiant qu'ils qualifient de nécessaire exercice de vérification de la bonne adéquation entre les formes de la vie religieuse et la finalité missionnaire de l'Ordre. Ils partagent la sensibilité des étudiants et souhaitent que les dominicains adoptent un mode de vie inséré dans le monde. Nicolas Rettenbach, invité à assister à une assemblée générale (AG), légitime publiquement la contestation étudiante: «Ce que vous avez engagé me paraît fondamentalement bon ${ }^{7}$. » Il autorise les assemblées à se poursuivre et les lecteurs de leur côté y participent activement. Le provincial convoque ensuite les prieurs de la Province les 12 et 13 juin 1968 afin qu'ils écoutent eux-aussi les frères étudiants. Il confère alors une autorité à la parole étudiante et corollairement donne une dimension provinciale à la contestation qui jusqu'alors restait limitée au seul Saulchoir.

Alors qu'un chapitre provincial ${ }^{8}$ est prévu en juillet 1969 pour réaliser « l'aggiornamento » de la Province demandée par le décret Perfectae Caritatis du concile Vatican II, le provincial décide de modifier le processus de réforme en y intégrant les frères étudiants. Dans l'ordre dominicain, les Constitutions organisent une forme de démocratie capacitaire. Pour y participer il faut avoir achevé ses études, être habilité à entendre les confessions, avoir au moins neuf ans de profession et être assigné légalement à un couvent. Les frères étudiants sont constitutionnellement exclus du pouvoir. Or Rettenbach va choisir de les intégrer à la réforme de la Province au même titre que les religieux plus âgés. À cette fin, il fait accepter aux prieurs la

\footnotetext{
${ }^{6}$ Yann Raison du Cleuziou, De la contemplation à la contestation, socio-histoire de la politisation de la Province dominicaine de France, thèse de doctorat de science politique, université Paris I, 2008.

${ }^{7}$ Archives de la province dominicaine de France (désormais APDF), IV, 202, 23, 4, Compte rendu de l'Assemblée du 24 mai 1968.

8 Assemblée qui réunit des membres élus et de droit qui représentent la Province et décident de ses orientations pour une période de trois ans. Le chapitre provincial est l'organe de gouvernement de la Province. Le provincial est élu lors de ce chapitre et applique les orientations qui y sont votées.
} 
Yann Raison du Cleuziou, «Éclatement du régime de l'autorité et dérégulation des relations de pouvoir : la Province dominicaine de France après Mai 68 », Histoire@Politique. Politique, culture, société, $n^{\circ} 18$, septembre-décembre 2012 [en ligne, www.histoire-politique.fr]

tenue d'assises de la Province en juillet 1969 juste avant le chapitre ${ }^{9}$. Elles seront une instance consultative représentant l'ensemble de la Province et non plus ceux qui, selon les Constitutions, ont droit à une représentation au chapitre provincial. Elles devront exprimer les orientations de la Province souhaitées pour les années à venir. Pour que les assises répondent effectivement aux attentes des frères, un long protocole de préparation est mis en place. L'ordre du jour des assises doit être établi à travers une consultation de l'intégralité de la Province, jeunes inclus bien sûr. Enfin, sur la question de la formation des jeunes dominicains, il décide de constituer une commission qui aura pour tâche de présenter un projet de réforme des études qui sera soumis aux membres de la communauté du Saulchoir réunis en AG pour l'occasion.

Ces décisions provinciales transforment ce qui se passe en mai 1968 en événement exceptionnel qui justifie un processus de réforme inédit ${ }^{10}$. Elles ouvrent une période qui est intéressante parce qu'elle permet d'étudier comment la Province de France va entrer dans une crise profonde qui durera jusqu'à la fin des années 1970. Une crise caractérisée, comme souvent dans l'Église, par un conflit d'interprétation sur les causes même de la crise, et par conséquent par la fin d'une reconnaissance partagée des normes de l'autorité au sein de l'Ordre ${ }^{11}$. Sans revenir sur les causes de cette crise, le caractère générationnel des groupes qui s'opposent, ou la trajectoire des réformateurs de l'Ordre, sujets traités dans d'autres articles ${ }^{12}$, nous voudrions ici nous attarder sur la dérégulation de l'institution entre mai 1968 et juillet 1969. Les relations de pouvoir se dérégulent parce que leur forme instituée a été modifiée d'une manière qui paraît illégitime à une partie des frères. Ils entrent alors en dissidence. C'est-à-dire qu'ils contestent les tenants de l'autorité au nom des principes que ces derniers devraient faire respecter. Leur désobéissance est une manifestation de leur fidélité et une dénonciation de l'infidélité de ceux qui s'aventurent dans le processus de réforme tel qu'il est organisé13. Dans ce contexte de flottement des fondements de l'autorité, les religieux s'affrontent de manière informelle en constituant des groupes de pression dans la finalité d'imposer la norme de ce qui doit faire autorité(I). La qualification des pratiques licites ne fait plus l'objet d'une reconnaissance unanime et devient un enjeu d'affrontement (II).

\footnotetext{
9 J ean-Pierre J ossua, « Le Saulchoir : une formation théologique replacée dans son histoire, (19561965), Cristianesimo nella storia XIV, février 1993, p. 99-124.

10 Yann Raison du Cleuziou, « La Province dominicaine de France et Mai 68 : genèse de la mise en crise d'un ordre religieux », dans J ean-François Gallinier-Pallerola, Augustin Laffay, Bernard Minvielle (dir.), L'Église de France après Vatican II, actes du colloque des 16 et 17 octobre 2009, Paris, Parole et Silence, 2011, p. 277-310.

${ }^{11}$ Jacques Lagroye, La vérité dans l'Église catholique. Contestations et restauration d'un régime d'autorité, Paris, Belin, 2006, chap. 1.

12 Yann Raison du Cleuziou, "Quand une génération en cache une autre. Différenciations générationnelles et mobilisations réformatrices dans la Province dominicaine de France autour de Mai 68 », Politix, vol. 24, n 96, 2011, p. 115-138.

${ }_{13}$ Sur la notion de dissidence dans l'ordre dominicain: Yann Raison du Cleuziou, " Des fidélités paradoxales : recomposition des appartenances et militantisme institutionnel dans une institution en crise », dans Michel Offerlé, J acques Lagroye (dir.), Sociologie de l'institution, Paris, Belin, 2011, p. 267290.
} 
Yann Raison du Cleuziou, «Éclatement du régime de l'autorité et dérégulation des relations de pouvoir: la Province dominicaine de France après Mai 68 », Histoire@Politique. Politique, culture, société, $n^{\circ} 18$, septembre-décembre 2012 [en ligne, www.histoire-politique.fr]

\section{Un conflit pour l'attribution du pouvoir de décision}

Dès le mois de juin 1968, plusieurs commissions sont créées pour élaborer des projets de réforme des études. On y retrouve les lecteurs les plus engagés dans la réforme des études et les frères étudiants les plus investis dans les AG du mois de mai. Les rédacteurs du projet sont donc moins qualifiés par leur fonction dans l'Ordre que par leur engagement dans la contestation. C'est pour un certain nombre de frères plus âgés une usurpation scandaleuse, une forme de coup de force, qui délégitime à leurs yeux le provincial qui l'autorise. L'existence de circuits de décisions parallèles à ceux prévus par les Constitutions achève de dissiper le respect dû aux autorités, il n'existe plus que des pouvoirs plus ou moins organisés en groupes concurrents. La désignation de l'arbitre qui doit trancher le conflit, parce qu'elle fixe les bornes du négociable, devient l'enjeu central de l'affrontement ${ }^{14}$. Pour les uns, c'est aux jeunes qui sont l'avenir de l'Ordre d'arbitrer ; pour les autres, ce sont aux Constitutions et à leur gardien, le Maître de l'Ordre, auxquels il faut recourir.

\section{La construction informelle de groupes de pression}

La période qui succède à Mai-Juin 1968 est caractérisée par la constitution de groupes de pression affinitaires au sein de l'ordre dominicain. Mais la capacité des religieux à se mobiliser collectivement n'est pas également partagée. Elle dépend de leur rapport au processus de réforme en cours.

Les jeunes lecteurs et les étudiants s'approprient d'autant plus volontiers les commissions et les consultations que leur action collective précède Mai 68. Les jeunes lecteurs, comme J ean-Pierre J ossua, Patrick J acquemont ou Bernard Rey, ont en commun d'être nés au début des années 1930, d'avoir été marqués par les novations missionnaires de l'après-guerre et d'avoir poursuivi leurs études dans le contexte de sévère retour à l'orthopraxie contemplative qui succède à l'arrêt des prêtres-ouvriers en 1954. Ils bénéficieront d'une promotion très rapide en raison du concile Vatican II car ils apparaissent alors aptes à inventer de nouvelles manières de penser la théologie et de l'enseigner. En 1965, à peine leurs études achevées, ils sont nommés lecteurs au Saulchoir pour épauler Claude Geffré dans son projet d'aggiornamento de l'enseignement théologique. Ces jeunes lecteurs forment déjà une équipe de travail réformatrice bien avant 1968. La contestation de Mai sera pour eux l'occasion d'accélérer la mise à l'agenda des réformes dont ils sont porteurs.

Quand aux frères étudiants, ils composent une communauté à part au sein du couvent du Saulchoir, le «studentat», dirigée par un Père-Maître, Albert-Marie Besnard, en charge de leur formation spirituelle. Les activités du «studentat», comme les retraites de rentrée, leur donnent l'occasion de discuter ouvertement de la pertinence des formes de la vie religieuse bien avant 1968. Ils constituent donc un groupe de pression structuré et organisé et savent manipuler les techniques nécessaires pour se faire entendre. Des élus étudiants font office de porte-parole, des

14 J acques Lagroye, « Institution, autorité, innovation », dans J acques Palard (dir.), Le gouvernement de l’Eglise catholique : synodes et exercice du pouvoir, Paris, Cerf, 1997, p. 307-312. 
Yann Raison du Cleuziou, «Éclatement du régime de l'autorité et dérégulation des relations de pouvoir : la Province dominicaine de France après Mai 68 », Histoire@Politique. Politique, culture, société, $n^{\circ} 18$, septembre-décembre 2012 [en ligne, www.histoire-politique.fr]

enquêtes d'opinion sont fréquemment réalisées pour faire connaitre l'état d'esprit des étudiants au corps enseignant, enfin la réalisation de rapports au sein de commissions de travail est également fréquente. Les AG du mois de mai dériveront d'ailleurs d'une conférence spirituelle où Albert-Marie Besnard accuse violement les frères étudiants de n'être pas suffisamment autonomes dans leur vie religieuse. Ces derniers déploient alors tout leur savoir-faire pour démontrer le contraire.

Pour les membres des commissions de préparation du projet de réforme de la formation, la difficulté est double. Ils doivent à la fois donner des garanties du sérieux de leur travail aux pères de la Province qui leur sont opposés, pour limiter leur résistance, mais aussi être suffisamment radicaux dans leur projet pour ne pas perdre l'adhésion des frères étudiants. J ean-Pierre J ossua, lecteur de 38 ans qui prend la tête de la commission en charge de la réforme des études, craint par-dessus tout une division des entrepreneurs de réformes issus de la mouvance du mois de mai. Ses craintes sont justifiées lorsqu'en janvier 1969, quatre frères étudiants présentent une esquisse de contre-projet (désigné comme l'avant-projet II) à celui des commissions. Son contenu est très radical puisqu'il propose la suppression totale du couvent d'étude et la fin de l'enseignement de la théologie. Les sciences sociales s'y substitueront. Ainsi seront formés « d'authentiques hommes croyants, artisans de ce monde et prophètes du Royaume ${ }^{15} »$.

Ce projet inquiète les lecteurs de la commission de rédaction du projet de formation. Ils craignent que la diffusion de l'avant-projet II dans la Province ne suscite de violentes réactions qui menaceront par là-même l'aboutissement du projet soutenu par la commission. Ce serait pour J ossua, « une erreur politique considérable ${ }^{16}$ ».

« Il me semble donc absolument capital que les frères qui sont décidés à une évolution franche ne se divisent pas, fassent des efforts pour surmonter tout désaccord partiel et toute susceptibilité, s'entendent pour proposer des projets communs - même s'il y a des points qui restent en suspens - , n'affaiblissent pas par leurs divisions ou par des positions actuellement irrecevables des chances d'évolution ${ }^{17}$. »

Jean-Pierre Jossua, appuyé par un certain nombre de frères étudiants tente de concilier les projets. Finalement, en guise d'alliance stratégique, un des étudiants à l'origine du projet dissident sera intégré à la commission de rédaction. La perspective des AG donne le change aux étudiants insatisfaits en garantissant leur participation future au processus.

À leur opposé, les religieux rétifs à une évolution refusent de participer à ces instances de peur de les cautionner et s'en tiennent à des formes individuelles de protestation. Non invités à participer aux commissions, ils ne peuvent s'exprimer que lors des AG prévues pour adopter le projet de réforme. Les frères les plus âgés, qui dénient toute légitimité au travail de la commission et aux AG, ne s'expriment que par lettres, multipliées par une ronéo, qui passent de mains en mains entre frères «conservateurs ». Pour eux, une prise de parole dans l'arène de l'AG ou une participation aux commissions, même critique, cautionneraient un processus qu'ils

${ }^{15}$ APDF, IV, 202, 23, 9, Avant-projet II, rédigé par les frères Courcier, Girard, Gonon, Le Gal, non daté. 16 APDF, IV, 202, 23, 7b, Lettre de J ean-Pierre J ossua, datée du 25 janvier 1969.

${ }^{17}$ Ibidem. 
Yann Raison du Cleuziou, «Éclatement du régime de l'autorité et dérégulation des relations de pouvoir : la Province dominicaine de France après Mai 68 », Histoire@Politique. Politique, culture, société, $n^{\circ} 18$, septembre-décembre 2012 [en ligne, www.histoire-politique.fr]

estiment illégitime. Respectueux de la hiérarchie, les courriers qu'ils adressent à leurs supérieurs restent leur unique moyen d'expression. Pierre-Marie de Contenson, philologue âgé de 50 ans, membre de la commission léonine ${ }^{18}$, est alors le prieur du Saulchoir. Défenseur de l'objection de conscience au début des années 1960, il est à la fois ouvert à certaines requêtes des frères étudiants et opposé à la forme irrégulière de leur protestation. Il craint surtout que l'enseignement du thomisme ne se trouve marginalisé par les réformes et par le gout exclusif pour les sciences sociales qu'affichent les jeunes. En raison de sa responsabilité priorale, il reçoit un nombre important des missives de protestation des pères qui refusent la voie que prennent les réformes. Contenson invite ceux qui viennent se plaindre auprès de lui du processus en cours d'aller dire leur opinion aux AG et y compris d'y aller pour voter contre.

Reste que lors de la première AG des 15 et 16 février, soixante-dix-huit frères sont présents dont une minorité d’opposants déclarés. Malgré quelques amendements sommaires, le texte sur la formation est adopté dans son ensemble. Pour rappeler l'extrême relativité d'un mode de consultation qui est boycotté par une partie des pères, le 21 février, Pierre-Marie de Contenson demande aux pères du Saulchoir de lui communiquer par écrit leur position sur le projet de la commission. Il désire les connaître en vue du conseil provincial du 6 mars où la question de la formation dans la Province sera abordée. Cette démarche perçue comme une stratégie de déni de la légitimité des AG est dénoncée par les membres de la commission. Contenson estime qu'il est juste dans son rôle de prieur quand il se soucie de faire entendre l'opinion des silencieux dont il reçoit les courriers. Il prétend que la consultation n'est pas un référendum car sans portée normative, qu'elle doit juste lui permettre de mieux représenter le couvent au conseil provincial ${ }^{19}$. Mais ses lettres ultérieures montrent qu'il n'en pense pas moins. Sur les trente-neuf réponses obtenues par Contenson : vingt sont radicalement contre; deux défavorables avec réserves; deux ne se prononcent pas; neuf sont radicalement pour; cinq favorables avec réserve. Les jeunes pères répondent collectivement par une lettre d'Antoine Lion, et affirment leur soutien au projet ${ }^{20}$. Tous les frères qui ont plus de 42 ans sont contre et ceux qui ont moins de 42 sont pour ${ }^{21}$.

Malgré la stratégie de Contenson, ces critiques restent marginales parce que leurs auteurs refusent d'intégrer le processus officiel d'élaboration de la réforme. $\mathrm{Au}$ contraire les frères étudiants s'en saisissent et y dominent. Le 25 mars, une nouvelle AG réunit la communauté du Saulchoir pour voter le nouveau texte où ont été intégrés les amendements de la précédente session. Le texte est adopté par 47 « oui » contre 21 « non ». Il est précisé dans le compte-rendu de l'AG que soixante-huit frères étaient présents sur cent frères qui habitent le couvent. Les vingt-cinq religieux qui ont le statut « extra-conventum », c'est-à-dire qui dépendent du prieur conventuel sans pour autant habiter le couvent, ne sont donc pas intégrés dans le total des cent. Il est noté qu'aucun n'a d'ailleurs voté. Le 29 mars, Pierre-Marie de Contenson diffuse une note précisant que le total de la communauté du Saulchoir est de cent

${ }^{18}$ Groupe de chercheurs dominicains en charge de l'édition critique des textes de saint Thomas d'Aquin.

${ }^{19} \mathrm{APDF}, \mathrm{IV}, 202,23,8$, Réponse de Pierre-Marie de Contenson à Patrick J acquemont.

${ }^{20}$ APDF, IV, 202, 23, 8, Lettre d'Antoine Lion à Pierre-Marie de Contenson, datée du 2 mars 1969.

${ }^{21}$ APDF, V, 651, 24, Lettre de Pierre-Marie de Contenson à Anicet Fernandez, datée du 14 mars 1969. 
Yann Raison du Cleuziou, «Éclatement du régime de l'autorité et dérégulation des relations de pouvoir : la Province dominicaine de France après Mai 68 », Histoire@Politique. Politique, culture, société, $n^{\circ} 18$, septembre-décembre 2012 [en ligne, www.histoire-politique.fr]

vingt-sept frères (il compte les extra-conventum) et sous-entend, en ironisant par des guillemets sur le terme "Assemblée générale », que les quarante-sept voix qui ont approuvé le projet des commissions sont minoritaires car l'AG n'est pas représentative 22 .

Mais ces calculs ne sont pas pris en compte par la partie adverse parce que les religieux dont Contenson tente de rendre visible la colère, ne sont pas considérés comme compétents pour réformer les études. Selon de Contenson, le provincial est juge et partie. Ayant autorisé le processus d'élaboration du projet de réforme des études, il ne peut que reconnaître les travaux qui en sont l'aboutissement. Les frères en réaction ne souhaitent effectivement pas élaborer un autre projet mais défendre ce qui existe. Ils se marginalisent en refusant de jouer le jeu de la réforme. Leurs protestations restent atomisées, contraintes à rester des échanges de lettres de personnes à personnes.

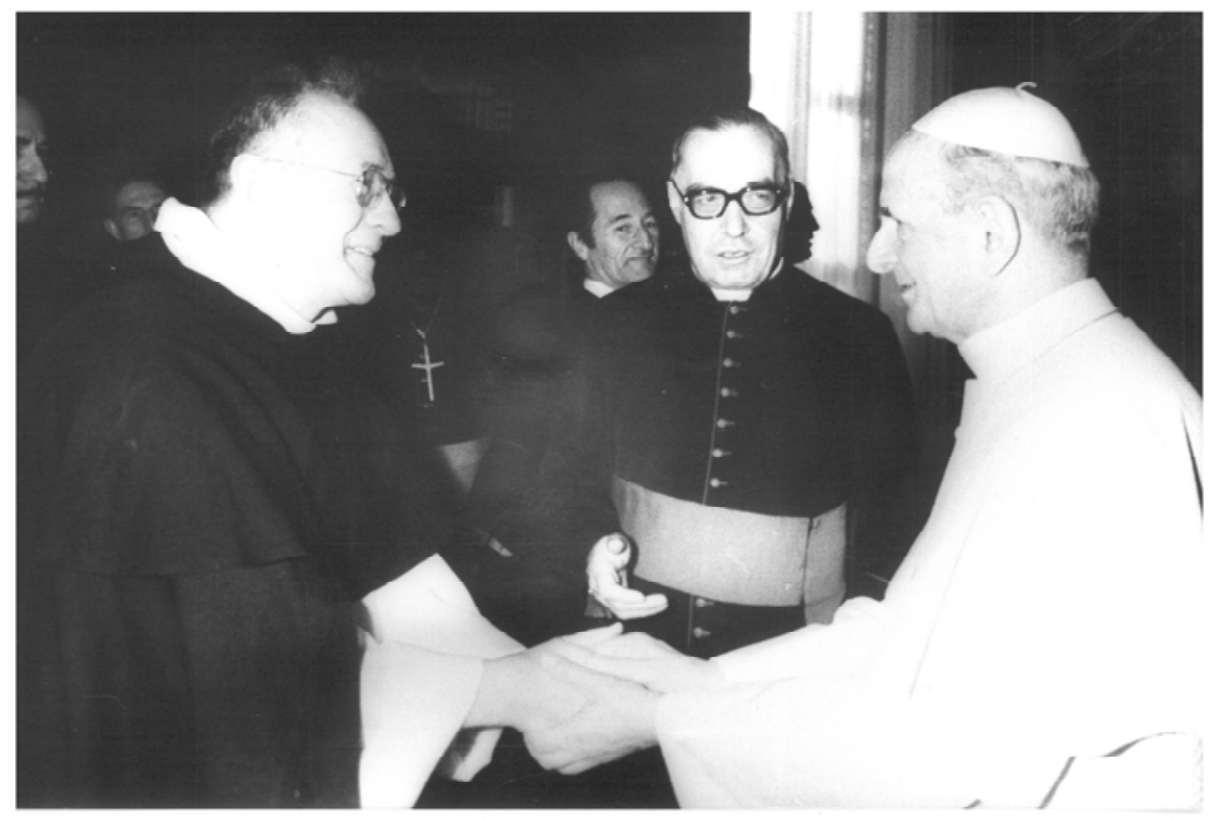

Pierre-Marie de Contenson avec Paul VI. @ ( Famille de Contenson

\section{Des légitimités concurrentes}

Pour légitimer ces procédures exceptionnelles, Nicolas Rettenbach a mobilisé un argument générationnel. Il affirme que l'afflux de vocations dominicaines risque de se tarir si des réformes ne viennent pas stimuler l'adhésion des jeunes générations. Dans

22 APDF, IV, 202, 23, 8, Résultats chiffrés concernant le fameux vote organisé au Saulchoir en "Assemblée générale" et qui a pesé lourdement sur l'avenir du Collège, rédigés par Pierre-Marie de Contenson, 29 mars 1969. 
Yann Raison du Cleuziou, «Éclatement du régime de l'autorité et dérégulation des relations de pouvoir : la Province dominicaine de France après Mai 68 », Histoire@Politique. Politique, culture, société, $n^{\circ} 18$, septembre-décembre 2012 [en ligne, www.histoire-politique.fr]

un contexte marqué par la prise de conscience de la crise des vocations ${ }^{23}$, l'argument a du poids. Par ailleurs, la pensée du provincial et de l'équipe de lecteurs, âgés d'une trentaine d'années, qui soutient l'initiative de la réforme du Saulchoir, est caractérisée par une certaine juvénophilie. Certains de vivre un grand tournant de la civilisation occidentale, une mutation profonde de la société, ils prennent très au sérieux les revendications des jeunes religieux. Elles témoignent selon eux des formes de l'attente religieuse qui naissent avec la société nouvelle.

Nicolas Rettenbach peut être qualifié de «professionnel » de la jeunesse en raison des missions successives qui furent les siennes dans l'ordre dominicain. Né en 1910, il est ordonné en 1935 et après une charge d'aumônier étudiant à Lille il est nommé Père-Maître des frères étudiants en décembre 1940. Sa pédagogie est centrée sur l'Évangile et il fait de la vie du Christ l'exemple exclusif par rapport auquel les jeunes religieux doivent penser leur action. Cette relativisation des normes de la vie religieuse dominicaine, et tout spécialement des observances monastiques, justifiera son changement de fonction en août 1955 alors que la Province de France est soumise au contrôle étroit du maître de l'Ordre en raison du soutien de ses théologiens aux prêtres-ouvriers ${ }^{24}$. Il deviendra ensuite aumônier des facultés de droit de Paris au Centre Saint-Yves. Très marqué par la pédagogie de l'Action catholique et par l'action des prêtres-ouvriers, il devient prêtre-étudiant pour partager les conditions de vie de ses ouailles. Par ailleurs, dans les instances de la Province, au côté des jeunes générations dominicaines qu'il a formées, il défend la possibilité dans l'Ordre d'un mode de vie non conventuel où les religieux pourraient partager la vie ordinaire des incroyants par le travail, la vie en appartement et les engagements sociaux et politiques. Les frères entrés dans l'Ordre depuis 1940 deviennent majoritaires dans le corps électoral de la Province en 1965. En 1967, ils élisent Nicolas Rettenbach à la charge de provincial. Il arrive à cette charge dans un moment important car le décret Perfectae Caritatis adopté lors du concile Vatican II invite les ordres religieux à réviser leurs formes de vie afin que leur témoignage soit plus compréhensible pour ceux qui sont en dehors de l'Église. Nicolas Rettenbach place les frères étudiants au coeur de ce processus. Selon lui, les normes de la vie religieuse doivent être « réinventées » en fonction de leurs aspirations parce qu'elle témoigne des attentes du monde. Il défendra cette idée lors du congrès des provinciaux dominicain à Rome en 1967: « Les plus jeunes de nos frères, qui sont l'espoir de l'Ordre, posent certaines questions [...], par ces questions ils sont d'ailleurs très représentatifs des hommes d'aujourd'hui, à qui nous sommes responsables de la Parole de Dieu ${ }^{25}$. » Cette interprétation de la jeunesse permet de renverser la hiérarchie de l'autorité dans la Province.

Cette juvénophilie confortée par l'expérience de Mai 68 est donc au coeur des justifications que le provincial déploie pour légitimer les procédures exceptionnelles que sont les diverses consultations provinciales, les commissions, les AG et les

\footnotetext{
${ }^{23}$ Denis Pelletier, La crise catholique, religion, société, politique en France, Paris, Payot, 2002, p. 50 et suiv.

24 François Leprieur, Quand Rome condamne, dominicains et prêtres-ouvriers, Paris, Plon, 1989.

25 APDF, III, B, 78, b, 17, Allocution de Nicolas Rettenbach au congrès des Provinciaux à Rome, 4 septembre 1967.
} 
Yann Raison du Cleuziou, «Éclatement du régime de l'autorité et dérégulation des relations de pouvoir : la Province dominicaine de France après Mai 68 », Histoire@Politique. Politique, culture, société, $n^{\circ} 18$, septembre-décembre 2012 [en ligne, www.histoire-politique.fr]

Assises de la Province. Les jeunes lecteurs mobilisent également cette rhétorique. L'un d'eux dénoncera les manœuvres de Pierre-Marie de Contenson parce qu'elles font intervenir des religieux incompétents pour traiter des réformes en raison de leur âge avancé. Il leur oppose ceux « dont l'avenir - et avec celui de la Province - est en question ici ${ }^{26} »$. C'est là un argument qui permet d'esquiver le critère de la représentativité et de la compétence au nom d'un critère de génération. L'argument est repris au sein de nombreux couvents par de nombreux pères d'une quarantaine d'années qui soutiennent les aspirations des frères étudiants parce qu'elles convergent avec leur propres conceptions de la refonte de la vie religieuse. «Qu'une génération légifère pour elle-même, voilà qui semble tout à fait naturel ; mais qu'elle transpose à celle qui la suit ses propres normes de fidélité, et ses formes de présence au temps qui passe, voilà qui, à mon avis, n'est guère sage et, en tout cas, est inacceptable ${ }^{27}$ » écrit le père Jourdain Lauchard au père Spitz qui dénonce les réformes.

L'argument que mobilisent les religieux attachés au mode de formation existant et qui refusent le projet de réforme est symétriquement inverse. Ils dénient toute légitimité aux jeunes religieux pour définir la vocation dominicaine. Le théologien Yves Congar se plaint par exemple que des frères qui ont peu d'expérience religieuse aient voix égale avec ceux qui ont quarante cinq années de vie religieuse ${ }^{28}$. Le père Dognin estime le projet utopique et ajoute aussitôt « ce qui n'a rien d'étonnant quand on sait que ce sont des "adolescents" qui l'ont élaboré». Il formera des « amateurs 29 » selon lui. Jacques Le Tilly, prieur du couvent de l'Annonciation demande par lettre à Nicolas Rettenbach de respecter les «anciens » qui « ont le droit de vieillir et de mourir tels qu'ils ont vécu toute leur vie». Tout en mettant en avant ses quarante-huit années de profession religieuse, il affirme que la rénovation entreprise selon les volontés des plus jeunes ne peut qu'être illégitime :

« Il ne conviendrait pas de proclamer les droits de certains à 'vivre leur vie' avant qu'ils n'aient eu le temps de savoir ce qu'est la vie dominicaine et comment elle a été vécue par d'authentiques apôtres. Formulée et décidée sous la pression de 'groupes d'action', sans l'approbation et même sans l'avis et l'information de tous, sans possibilité d'expression pour chacun et sans espérance d'être entendu, une telle 'rénovation' risquerait d'apparaître arbitraire, et engendrerait bien des souffrances : l'avenir de beaucoup risque d'être vécu dans une perpétuelle interrogation de notre esprit et une immense tristesse de notre coeur ${ }^{30}$. »

Le malaise des jeunes témoigne moins selon lui de l'inadaptation de l'Ordre, que de leur infidélité. La disqualification qu'estiment subir ces religieux rétifs à la réforme aboutit à exacerber leur exaspération. Après avoir tenté de contester la représentativité des AG puis la compétence des entrepreneurs de réforme, PierreMarie de Contenson finira par diffuser une protestation dont le ton n'a plus rien de

\footnotetext{
${ }^{26}$ APDF, IV, 202, 23, 8, Lettre à Pierre-Marie de Contenson, datée du 27 février 1969.

27 APDF, 202, 23, 8, Réponse au texte manifeste envoyé par le père Spitz, rédigée par Jourdain Lauchard, non datée.

${ }^{28}$ APDF, IV, 202, 23, 8, Lettre de Yves Congar à Pierre-Marie de Contenson, datée du 22 février 1969.

${ }^{29}$ APDF, IV , 202, 23, 7, Lettre du père Dognin à Pierre-Marie de Contenson, février 1969.

30 APDF, III G, 50, 101, Lettre de J acques Le Tilly à Nicolas Rettenbach, datée du 25 août 1968, p. 2.
} 
Yann Raison du Cleuziou, «Éclatement du régime de l'autorité et dérégulation des relations de pouvoir : la Province dominicaine de France après Mai 68 », Histoire@Politique. Politique, culture, société, $n^{\circ} 18$, septembre-décembre 2012 [en ligne, www.histoire-politique.fr]

policé. Il dénonce «un régime d’assemblée» qui implique « un nivellement démocratique absolu ignorant toute référence à l'autorité soit d'une hiérarchie religieuse, soit d'une compétence technique, soit d'une expérience, soit d'une tradition, et par lequel ipso facto la majorité se trouvait du côté de ceux qui avaient le moins de compétence, d'expérience et de sens religieux et où les meneurs révolutionnaires et les tribuns doctrinaires devaient inévitablement jouer un rôle primordial et ont de fait dès le début fait régner un climat de relative intolérance et de surenchères outrancières ${ }^{31} »$. On aurait tort de lire ici une simple manifestation d'antidémocratisme, en creux, Contenson fait l'apologie de ce qui est pour lui la seule démocratie légitime dans l'ordre dominicain : une démocratie capacitaire où la participation aux décisions dépend de la compétence et de l'ancienneté du temps de profession.

Réduits au silence en raison de leur déférence aux règles de l'ordre dominicain, les religieux opposés au projet de réforme des études, ne peuvent que se retourner contre le titulaire de l'autorité provinciale qui selon eux, légitime et autorise des pratiques illicites.

\section{Les frontières de l’obéissance : un enjeu disputé}

La période qui succède à Mai-Juin 1968 est caractérisée par la fin d'une reconnaissance unanime des principes qui fondent l'autorité au sein de l'ordre dominicain. Or l'autorité procure au groupe des références communes, sous-tend l'élaboration du droit et du règlement, et s'objective dans une hiérarchie et des rôles institués qui, finalement, fondent, en le mesurant, le respect dû à chacun. Après les décisions de Nicolas Rettenbach, les repères qui stabilisaient la configuration institutionnelle font place à un doute généralisé qui se traduit en pratique par une concurrence dérégulée pour la maîtrise du pouvoir de définir les rôles, pratiques et savoirs légitimes. Cela se traduit tout spécialement dans les pratiques d'obéissance. Avec la contestation de l'autorité dans la Province, les critères de ce qui rend l'obéissance légitime ou non deviennent flous et leur définition est un enjeu de lutte.

\section{Une disqualification de l'autorité provinciale}

In fine, les opposants à la réforme en viennent à reporter en amont leurs critiques. Ils remettent en cause celui qui l'a autorisée, le provincial Nicolas Rettenbach. Le père Lelong l'accuse ainsi directement de favoriser la destruction de l'ordre dominicain en donnant le pouvoir aux jeunes :
«La consultation des jeunes est une aberration, les jeunes n'ont pas à réformer l'Ordre: ils ont à exister d'abord, à entrer dans l'esprit de l'Ordre qui ne les a pas attendus pour être ce qu'il est. Quand on en sait plus quel est précisément son but, ou quand on conteste sa nature, on n'y entre pas ou si l'on y est, on en sort. Personne n'est obligé d'être dominicain ou catholique, mais ceux qui ont fait profession religieuse ou profession de foi ont le droit et le devoir de dénoncer ceux qui, de

\footnotetext{
${ }^{31} \mathrm{APDF}, \mathrm{V}, 651,24$, Note sur le Projet de réforme de la formation élaboré au Saulchoir d'Étiolles, PierreMarie de Contenson, non daté.
} 
Yann Raison du Cleuziou, «Éclatement du régime de l'autorité et dérégulation des relations de pouvoir : la Province dominicaine de France après Mai 68 », Histoire@Politique. Politique, culture, société, $n^{\circ} 18$, septembre-décembre 2012 [en ligne, www.histoire-politique.fr]

l'intérieur, détruisent l'Ordre ou l'Église, qui les a reçus. Et vous, chargé de veiller à l'intégrité d'un bien de famille qui ne vous appartient pas, vous les cautionnez et favorisez ainsi la débâcle ${ }^{32}$. »

La référence à la profession religieuse rappelle que les religieux en s'engageant dans l'ordre dominicain ont juré fidélité à ses Constitutions. À l'instar de Lelong, de nombreux pères revendiquent leur observance des Constitutions afin de mieux dénoncer la sollicitude de Rettenbach et des lecteurs à l'égard des jeunes religieux. Elle est pour eux une manifestation de démagogie ou de lâcheté. Le père Spitz dénonce les faveurs du provincial aux frères étudiants et aux lecteurs contestataires. Il les juge irrégulières : " C’est à croire qu'il faille être contestataire et de préférence hérétique pour avoir de nos jours quelqu'audience. » Si le provincial inverse la hiérarchie, l’Ordre est ébranlé :
«Vous vous plaignez d'être débordé par votre charge: comment ne le seriez-vous pas? Vous avez à conduire une Province Dominicaine hiérarchisée, et vous commencez par la transformer en un troupeau de moutons : demander à chacun ses desiderata, c'est aller nécessairement, en présence de cinq cents avis différents, vers l'émiettement. Vous allez être dépassé par les "enragés", qui veulent, ils le disent eux- mêmes, "casser la baraque". Encore une fois, le problème essentiel est celui de notre unité religieuse ${ }^{33}$. 》

Henri Féret formule une critique analogue. Selon lui, en accordant le même crédit à toutes les aspirations, le provincial déstructure l'Ordre. Il détruit la voie de conformation à un modèle qu'il devrait avoir soin de faire perdurer :
«C'est à chacun désormais qu'il revient d'être son propre formateur, dans la fidélité à son 'projet initial', voire aux valeurs évangéliques qui l'impressionnent le plus, mais en une indépendance pratique par rapport à une tradition cependant très spécifiée dans l'Église et dans l'histoire. La vie religieuse doit ainsi s'adapter désormais aux requêtes d'un chacun, et non plus l'inverse. Il est certes possible que la tradition proprement religieuse et spirituelle de l'Ordre ne convienne pas à tous : mais alors, que chacun cherche sa propre voie devant Dieu, mais ne "déstructure" pas cette tradition originale dans l'Église ${ }^{34}$. »

Yves Congar est très pessimiste car selon lui tout est déjà joué d'avance à cause d'un groupe de lecteurs qui oriente le Collège et de Rettenbach qui est partie prenante ${ }^{35}$. En définitive ce qui mobilise ces frères, c'est la peur d'assister à la destruction de l'ordre dominicain et l'imputation de la responsabilité de cette politique au provincial. Nicolas Rettenbach a désobéi à sa mission selon eux. Ils se sentent marginalisés et considérés comme des déviants en raison de leur fidélité à leurs voux. Constatant que l'autorité provinciale ne défend plus l'autorité des

\footnotetext{
32 APDF, IV, 202, 23, 8, Déclaration du père Lelong au père Rettenbach à l'occasion de la visite canonique du couvent de l'Annonciation, le 10 février 1969.

${ }^{33} \mathrm{APDF}$, III, J , 16, 3 Note du père Spitz à l'occasion de la visite canonique du 28 janvier 1968.

34 APDF, IV, 202, 23, 7, c, Annotations du « document de travail sur la formation des frères » paru en mai 1969, Henri-Marie Féret, 25/ 26 juin 1969, p. 9.

35 APDF, IV, 202, 23, 8, Lettre de Yves Congar à Pierre-Marie de Contenson, datée du 22 février 1969.
} 
Yann Raison du Cleuziou, «Éclatement du régime de l'autorité et dérégulation des relations de pouvoir : la Province dominicaine de France après Mai 68 », Histoire@Politique. Politique, culture, société, $n^{\circ} 18$, septembre-décembre 2012 [en ligne, www.histoire-politique.fr]

Constitutions, ces religieux n'ont plus qu'un seul recours pour tenter d'endiguer la réforme : Rome.

\section{La mise en concurrence des autorités}

Pour contrer la politique provinciale de Rettenbach, dont l'autorité est d'autant moins reconnue qu'il apparaît compromis, Pierre-Marie de Contenson et un certain nombre d'autres frères envoient des lettres alarmistes au Maître de l'Ordre, Anicet Fernandez, et lui demandent d'intervenir ${ }^{36}$. Ce dernier est attentif à ces courriers qui convergent avec sa propre appréciation de l'évolution de la Province de France. Le 21 avril 1969, il écrit à Nicolas Rettenbach pour lui signifier clairement sa défiance vis-à-vis des projets de réforme de la formation. Selon lui, le Saulchoir est un centre intellectuel indispensable à la Province et le supprimer serait une erreur dont les prochaines générations pâtiraient. Les réformes lui apparaissent de périlleuses aventures :

« Permettez-moi de vous le dire très simplement, mon cher Père, je ne puis croire que votre Province dont tant de religieux font, aujourd'hui même, honneur à l'Ordre dans la fidélité à saint Dominique et à la tradition du Père Lacordaire, accepte de se lancer du jour au lendemain sur des chemins aussi nouveaux et qui risquent fort de mener à l'aventure ${ }^{37}$. »

Il voit dans les oppositions manifestées au projet «un signe de santé et une promesse » de retour à l'ordre dans la Province. Mais cette lettre n'a pas un caractère public et seul son destinataire en connaît le contenu.

Au début du mois de mai 1969, Contenson se rend à Rome au prétexte d'affaires à régler pour la commission léonine. En fait, il souhaite seulement rencontrer le Maître de l'Ordre pour obtenir son soutien explicite contre les tendances réformistes au sein de la Province de France ${ }^{38}$. Cette initiative est encouragée par un certain nombre de frères dont Pie Régamey et André Duval ${ }^{39}$. Ils souhaitent que Fernandez publie une lettre rappelant que les assises et le chapitre provincial restent soumis aux Constitutions et qu'il veillera à faire appliquer cette règle fondamentale. Les plus radicaux mettent même en balance leur appartenance à l'Ordre. Selon eux, si l'Ordre n'était pas fidèle à lui-même, ils s'estimeraient libérés de leurs vœux.

Contenson obtient ce qu'il souhaite. Fernandez l'assure de son soutien et pour l'attester, lui montre la lettre qu'il a écrite à Rettenbach au mois d'avril. Il autorise le prieur à diffuser le contenu de sa lettre auprès des frères qui seraient inquiets. Par une lettre du 15 mai, Contenson envoie des copies de la lettre à onze frères qui avaient eux-mêmes écrit au Maître de l'Ordre et en attendaient une réponse ${ }^{40}$, mais le père de Rudder qui est destinataire de cet envoi la divulgue plus largement encore ${ }^{41}$. Le provincial, en apprenant que cette lettre est diffusée, accuse Contenson de ne pas

\footnotetext{
36 APDF, V, 651, 24, Lettre de Pierre-Marie de Contenson à Anicet Fernandez, datée du 14 mars 1969. ${ }^{37}$ APDF, IV, 202, 23, 9, Lettre d'Anicet Fernandez à Nicolas Rettenbach, datée du 21 avril 1969

38 APDF, V, 651, 25, Lettre de Pierre-Marie de Contenson à Anicet Fernandez, datée du 7 mai 1969.

39 APDF, V, 651, 25, Lettre d'André Duval à Pierre-Marie de Contenson, datée du 4 juin 1969 \& V, 651, 24, Lettre de Pierre-Marie de Contenson à André Duval, datée du 25 avril 1969.

${ }^{40}$ APDF, V, 651, 25, Lettre de Pierre-Marie de Contenson à Anicet Fernandez, datée du 28 juillet 1969.

${ }^{41}$ APDF, V, 651, 25, Lettre du père de Rudder à Nicolas Rettenbach, datée du 29 mai 1969.
} 
Yann Raison du Cleuziou, «Éclatement du régime de l'autorité et dérégulation des relations de pouvoir : la Province dominicaine de France après Mai 68 », Histoire@Politique. Politique, culture, société, $n^{\circ} 18$, septembre-décembre 2012 [en ligne, www.histoire-politique.fr]

respecter son autorité. Ce dernier répondra en retournant l'accusation car selon lui c'est Rettenbach qui sape tout ce qui fait autorité dans la Province :

« Pour ce qui est des procédés qui ruinent l'autorité. J'aurais souhaité que ce souci ait été présent en bien des occasions dans l'esprit de mes supérieurs, et par exemple du provincial de France quand, appelé par le prieur du Saulchoir, il a trouvé bon de tolérer et même d'encourager des assemblées générales ignorant toute autorité locale et toute considération d'appartenance différentielle à l'Ordre et ruinant par là même la structure démocratique du couvent pour y substituer un régime de démagogie incontrôlée ${ }^{42}$. 》

Le provincial publicisera l'affaire dans une lettre écrite à la Province. Il s'y justifie de n'avoir pas publié la lettre du Maître de l'Ordre en raison de son caractère privé et met nommément en cause le père deContenson et le père Spitz pour leurs manœuvres qui paraissent « suspecter la volonté de fidélité » à Saint Dominique des jeunes frères de la Province.

Au couvent du Saulchoir, l'autorité priorale de Contenson est ébranlée par l'affaire. Son recours à Rome apparait un geste déloyal. J acques Pohier lui écrit qu'il n'a plus aucune autorité morale sur la communauté43. Un autre jeune lecteur lui conseille de ne plus assister à aucune réunion pour éviter tout heurt et refuse dorénavant de collaborer avec lui ${ }^{44}$. D'autres lettres de jeunes lecteurs abondent dans le même sens $^{45}$. Plusieurs demandent au prieur de démissionner ${ }^{46}$. Au contraire, des lettres de Jean de Menasce, André Duval, Anicet Fernandez, du père Lelong et de JeanDominique Piollet, encouragent le prieur à ne pas céder. L'affaire, parce qu'elle oppose Contenson à Rettenbach, devient une occasion d'expression du clivage entre conservateurs et réformistes ${ }^{47}$. Deux conceptions de l'obéissance s'affrontent également. Pour les uns, les Constitutions priment le provincial, pour les autres c'est l'inverse.

Contenson préfère un temps ne pas démissionner de peur de paraître donner raison à ses adversaires. Il estime avoir obéi à un ordre du Maître général. D’ailleurs, ce dernier, par un télégramme du 3 juin, lui demande de ne pas quitter son poste, l'assure de son soutien, et minimise l'incident ${ }^{48}$. Le 8 juin, estimant ne plus avoir la confiance de sa communauté, Contenson démissionne finalement de sa charge de prieur du Saulchoir ${ }^{49}$. La demande est examinée lors du conseil provincial du 14 juin. Le père Congar estime qu'il est préférable que le père de Contenson conserve sa charge jusqu'au chapitre provincial conformément aux législations de l'Ordre. Les pères Chenu, Camelot, Carré soutiennent cette option. Malgré l'opposition d'un jeune lecteur très engagé dans la réforme des études, Rettenbach adoptera la position de

42 APDF, IV, 202, 23, 9, Lettre de Pierre-Marie de Contenson à Nicolas Rettenbach, datée du 20 mai 1969.

43 APDF, IV, 202, 23, 9, Lettre de Pierre-Marie de Contenson à J acques Pohier, datée du 2 juin 1969.

44 APDF, V, 651, 25, Lettre à Pierre-Marie de Contenson, datée du 31 mai 1969.

45 Cf, APDF, V, 651, 25.

${ }^{46}$ APDF, V, 651, 25, Lettre à Pierre-Marie de Contenson, datée du 6 juin 1969.

${ }^{47}$ Sur ce point voir les réactions conservées en APDF, V, 651, 25.

48 APDF, V, 651, 25, Télégramme d'Anicet Fernandez, daté du 3 juin 1969.

49 APDF, IV, 202, 23, 9, Lettre de Pierre-Marie de Contenson à Nicolas Rettenbach, datée du 8 juin 1969. 
Yann Raison du Cleuziou, «Éclatement du régime de l'autorité et dérégulation des relations de pouvoir : la Province dominicaine de France après Mai 68 », Histoire@Politique. Politique, culture, société, $n^{\circ} 18$, septembre-décembre 2012 [en ligne, www.histoire-politique.fr]

Congar et suggérera à Contenson de remettre sa démission au chapitre provincial 50 . Par ailleurs il pense sans doute que les frères opposés aux réformes prendraient la démission de Contenson comme une censure de leur courant au futur chapitre et il souhaite éviter d'attiser encore plus leur défiance envers les réformes ${ }^{51}$. Rettenbach veut éviter tout heurt susceptible de remettre en cause les Assises. Aucune partie de la Province ne doit être tentée de les boycotter, car de sa représentativité dépend sa légitimité.

Conscient que sa charge de prieur arrive à échéance, Contenson va profiter des deux mois qui lui restent pour user des moyens de résistance que sa fonction met à sa disposition. Lors des assises provinciales qui se déroulent du 30 juin au 5 juillet, il profite de son statut de membre de droit pour s'opposer de nouveau au projet de réforme de la formation. Il rédige une motion critiquant la représentativité de la commission d'élaboration du projet et suggère d'en créer une nouvelle composée de «représentants qualifiés » et soumise au Maître de l'Ordre afin d'assurer l'orthopraxie du projet final. La motion sera repoussée par 52 «non », alors que 24 frères ont voté « oui » et 12 supplémentaires « oui avec réserve » 52 . Les jeunes religieux sont surreprésentés dans la composition des Assises.

Le 9 juillet lors d'un conseil provincial élargi où sont réunis tous les supérieurs de la Province, le prieur du Saulchoir demande la démission de Rettenbach au motif qu'il divise la Province en se laissant conduire par « quelques religieux extrémistes ${ }^{53}$ ». Contenson échoue. Rettenbach est reconduit dans sa charge après un vote de confiance. Finalement, le 31juillet, à l'occasion du chapitre provincial, Contenson donne sa démission. Elle est acceptée. Pour ne pas prendre le risque d'un éclatement de la communauté du Saulchoir à l'occasion de l'élection du nouveau prieur, Rettenbach nomme Albert Raulin, vicaire provincial, pour le Saulchoir. Ce dernier était en mission à l'étranger et ne peut donc apparaitre lié à l'un ou à l'autre des groupes de dominicains qui se déchirent.

L'étude de la Province dominicaine de France montre la nécessité de contextualiser finement la mise en crise des institutions après Mai 68. Trop souvent la crise de l'autorité est imputée au contexte. Qu'il soit ecclésial avec le concile Vatican II, social avec la montée en puissance de la culture jeune durant les sixties, ou politique avec l'efflorescence des gauchismes en Mai-J uin 68. Force est de constater qu'aucune de ces causes ne suffit à expliquer ce qui se passe dans l'ordre dominicain en France durant les années 1970. La réalité est à la fois plus complexe et bien moins mécanique. Par exemple, rien n'est imposé par la « force de l'événement » Mai 68. Bien au contraire, dans la Province de France, ce qui va donner une force à la contestation étudiante, c'est la décision du provincial et la volonté des jeunes lecteurs d'en tirer parti pour bouleverser le processus de réforme mis en route par le concile Vatican II. Rétrospectivement, c'est en raison de ces décisions que Mai 68 est devenu

\footnotetext{
50 APDF, III, I, 6, Compte rendu de la séance du Conseil Provincial du 14 juin 1969.

${ }^{51} \mathrm{APDF}, \mathrm{V}, 651,25$, Lettre d'Yves Congar à Pierre-Marie de Contenson, datée du 7 juin 1969.

52 « Compte rendu intégral des Assises provinciales au Saulchoir », Provincialia, numéro spécial, 1969, p. 56.

53 APDF, V, 651, 25, Lettre de Pierre-Marie de Contenson au Maître de l’Ordre, datée du 7 juillet 1969.
} 
Yann Raison du Cleuziou, «Éclatement du régime de l'autorité et dérégulation des relations de pouvoir: la Province dominicaine de France après Mai 68 », Histoire@Politique. Politique, culture, société, $n^{\circ} 18$, septembre-décembre 2012 [en ligne, www.histoire-politique.fr]

un événement marquant de l'histoire de la Province dominicaine de France. L'exemple des dominicains permet donc de mesurer l'importance des stratégies de mobilisation et de contre-mobilisation déployées par les acteurs dans cette période. C'est d'elles dont dépend la mise en crise des institutions.

Dans la Province dominicaine de France, un autre point mérite d'être souligné. L'été 1968 est un moment de basculement de la domination religieuse. J usqu'alors, ce sont au sein des couvents de la Province, des religieux âgés d'une cinquantaine d'années qui se partagent les postes de responsabilité et imposent une certaine conception de l'orthopraxie dominicaine structurée autour des observances régulières et de l'étude. Après l'été, ces derniers se trouvent marginalisés de fait par les procédures inédites de réforme introduite par le provincial. À ce titre, on peut écrire qu'il s'opère un renversement de la déviance à l'été 1968. Si les frères étudiants contestent l'autorité des normes de la vie religieuse jusqu'en Mai 68, la politique du provincial fait d'eux de loyaux sujets dans la période qui suit. Ils passent de la contestation à la réforme en acceptant d'entrer dans les formes de négociation et de décision autorisées. Au contraire, ce sont les tenants de l'autorité avant Mai 68 qui se retrouvent dans une quasi-déviance parce qu'ils estiment que le provincial abuse de ses fonctions.

Cet engagement perçu comme une vacance de l'autorité par les religieux les plus conservateurs dérégule le rapport de force au sein de la Province. Les formes instituées de la démocratie dominicaine sont démonétisées par la création de procédure de consultations inédites. La fidélité à la vocation dominicaine se pluralise. Elle est également revendiquée par tous, mais ses formes deviennent antagonistes, parce que le référent qui la fonde repose maintenant dans une décision personnelle : la recherche d'authenticité personnelle se substitue à celle de conformité collective. Pour imposer une définition de l'Ordre comme la seule légitime, les frères ont moins recours à l'autorité qu'à des stratégies de pouvoir.

Les multiples pratiques de dissidence possibles en offrent tout un éventail. De la dénonciation ouverte à l'envoi de lettre de dénonciation au Maître de l'Ordre, d'un vote de protestation au boycott des assemblées. Ces diverses voies de la contestation ne sont jamais neutres et elles objectivent la manière dont chacun comprend son rôle. Pierre-Marie de Contenson use de toutes les ressources que sa responsabilité de prieur autorise et il légitime ses protestations au nom des membres silencieux de son couvent. Le père Lelong en chroniqueur à la plume alerte, publiera dans L'Aurore des articles violents pour dénoncer la dérive de la Province. Bien d'autres boycotteront silencieusement la réforme et s'attacheront à manifester une fidélité minutieuse à la vie régulière comme pour mieux dénoncer la vacuité du reste. Quoi qu'il en soit les multiples formes de la dissidence apportent beaucoup de renseignements sur les cultures collectives de la protestation dans l'Église et sur leurs ressorts individuels. C'est un sujet qui reste en grande partie à explorer.

Après un an d'affrontements aigus, en juillet 1969, le chapitre provincial reconnaîtra légitime le pluralisme des interprétations de la vocation dominicaine. La vie en communautés de bases engagées dans le monde devient aussi légitime que la vie en couvent. L'Ordre éclatera alors en de multiples modalités d'appartenance et se recomposera en communautés affinitaires et concurrentes dans leur prétention à 
Yann Raison du Cleuziou, «Éclatement du régime de l'autorité et dérégulation des relations de pouvoir : la Province dominicaine de France après Mai 68 », Histoire@Politique. Politique, culture, société, $n^{\circ} 18$, septembre-décembre 2012 [en ligne, www.histoire-politique.fr]

instituer la vie religieuse dominicaine légitime par leurs pratiques. Le pluralisme n'est pas imputable à «l'avènement de l'individualisme moderne », comme l'affirment bien des sociologues. Dans l'ordre dominicain, sa reconnaissance est alors le seul moyen de concilier l'hétérogénéité croissante des interprétations de la véritable vocation dominicaine avec le maintien d'un semblant d'unité. Comme l'a écrit Étienne Fouilloux : «Qu'est-ce, en effet, que le pluralisme, sinon l'aveu implicite du brouillard qui noie une vérité aux contours naguère si précis ${ }^{54}$ ? »

\title{
L'auteur
}

Yann Raison du Cleuziou est maître de conférences en science politique à l'université Montesquieu Bordeaux IV et membre du Centre Émile Durkheim (UMR CNRS 5116). Sa thèse sur les transformations de la vie religieuse dans l'Ordre dominicain entre l'après-guerre et la fin des années 1970 a reçu le Prix Richelieu en science politique de la Chancellerie des universités de Paris en 2009. Ses recherches actuelles portent sur les mobilisations politiques dans le monde rural, la politisation des pratiques religieuses et l'histoire contemporaine de l’Église catholique.

\section{Résumé}

En Mai-Juin 1968, les frères étudiants de la Province dominicaine de France dénoncent le caractère suranné des formes instituées de la vie religieuse. Cette contestation est soutenue par le provincial et par la jeune génération des enseignants en théologie. Un processus inédit de réforme est engagé sous leur autorité : les frères étudiants sont associés au même titre que les religieux plus âgés à la réforme de la vie religieuse de l'Ordre. Cet engagement, parce qu'il est considéré illégitime par une partie des religieux, provoque un discrédit du provincial et une perte de crédibilité des règles de fonctionnement de l'institution dominicaine. Faute d'une reconnaissance partagée du fondement de l'autorité dans l'Ordre, les rapports de pouvoir se dérégulent et des groupes de pression affinitaires se constituent et entrent en lutte.

Mots clés : crise, Mai 68, autorité, dominicains, contestation, ordre religieux.

\begin{abstract}
"Burst of authority and deregulation of power relationships : the French Province of the Dominican Order after May 68"

In May-J une 1968, the brothers students of the French Province of the Dominican order denounce the antiquated character of established forms of religious life. This protest is supported by the Provincial and the Younger Generation of Teachers in Theology. An unprecedented process of reform is committed under their authority: the brothers students are involved as well as their older brothers in the reform process of the religious life of the Order. This commitment, because it is considered as illegimate by some monks, causes discredit to the Provincial and the loss of credibility of the operating rules of the institution. Lacking a shared recognition of the basis of the authority in the Order, peer pressure groups are formed and come into conflict.
\end{abstract}

Key words : Crisis, May 68, authority, dominican brothers, protest, religious order.

Pour citer cet article : Yann Raison du Cleuziou, « Éclatement du régime de l'autorité et dérégulation des relations de pouvoir : la Province dominicaine de

\footnotetext{
54 Étienne Fouilloux, op. cit., p. 344.
} 
Yann Raison du Cleuziou, «Éclatement du régime de l’autorité et dérégulation des relations de pouvoir : la Province dominicaine de France après Mai 68 », Histoire@Politique. Politique, culture, société, $n^{\circ} 18$, septembre-décembre 2012 [en ligne, www.histoire-politique.fr]

France après Mai 68 », Histoire@Politique. Politique, culture, société, n 18 , septembre-décembre 2012 [en ligne, www.histoire-politique.fr] 